\title{
Predictive toxicology and ecotoxicology within the ANTIOPES network
}

\author{
Emmanuel Lemazurier • Francelyne Marano
}

Received: 28 December 2012 / Accepted: 7 January 2013 /Published online: 27 January 2013

(C) Springer-Verlag Berlin Heidelberg 2013

ANTIOPES is a French National Network which ambitions to answer main needs for REACH implementation while pursuing its action under partnership research and valorization of technical, scientific, and social tools.

ANTIOPES aims then to:

- Create a knowledge and initiative space in order to structure innovation at national and European scale,

- Bring answers to new requirements in chemical sector and propose a valorization space of toxicology research, mainly for new developed tools,

- Add value to transpositions between scales and living models, to exceed conceptual, technological, and methodological limitations.

The ANTIOPES network has organized on March 19th and 20th 2012 a Symposium in Avignon which has been attended by 150 participants. The scientific meeting was focused on one of its main project, the predictive ecotoxicology and toxicology, in order to produce a comprehensive update on technical and scientific innovations required for main challenges of chemical safety assessment: nanoparticles mainly on barriers and compartmentalization, rele-

Responsible editor: Philippe Garrigues

\section{E. Lemazurier $(\square)$}

Institut National de l'EnviRonnement industriel et des rISques (INERIS), Parc technologique Alata PB2,

60550, Verneuil En Halatte, France

e-mail: Emmanuel.LEMAZURIER@ineris.fr

\section{F. Marano}

Université Paris Diderot-Paris 7

Laboratoire des réponses moléculaires et cellulaires aux xénobiotiques (RMCX) Unité de biologie fonctionnelle et adaptative (BFA) CNRS EAC 4413,

4 rue Marie-Andrée Lagroua Weill-Hallé,

75205, Paris, Cedex 13, France

e-mail: marano@univ-paris-diderot.fr vance of biomarkers in endocrine disruption, and multiple stressor and vulnerability problem. In a last part of this scientific meeting, certification and translational research aspects to regulation have been highlighted. Underlying the symposium, specific projects developed in the frame of the implementation of the Operational Research Comity of the French Grenelle of Environment have been presented.

In this special issue, original data from the selected projects in predictive toxicology and ecotoxicology are presented. The first three articles are dealing with concepts applied to the above subject. Bado-Nilles et al. present a study dealing with lysosomal membrane stability. This work proposes two more rapid, efficient, and sensitive sample analyses using flow cytometry method. Lysosomal presence and lysosomal membrane integrity (LMI) were evaluated on the three-spined stickleback, Gasterosteus aculeatus (L.), a well-described model fish species for aquatic ecotoxicology studies. The designed immunomarkers were shown clearly modulated by pollutants and their variations seemed to be correlated with leucocyte mortality. Thus, from a practical point of view, lysosomal presence and LMI may provide novel and efficient means of evaluating immune capacities and indicating the toxic effects of environmental pollution. The next article from Zalko et al. is exploring complex mixtures of contaminants present in environment and in food chain with potential adverse effects on human health and wildlife. These authors explored the possibility to use Nuclear Receptor-based affinity columns to characterize the presence of bioactive molecules in environmental complex mixtures (mainly endocrine disruptor compounds). Estrogen Receptor $\alpha$-based affinity columns were used to trap and purify estrogenic substances present in surface sediment samples collected in a French river under mixed anthropogenic pressure. Biological, biochemical, and analytical approaches were combined to characterize the structure of ligands retained on columns and demonstrate the presence of known active molecules such as bisphenol $\mathrm{A}$ and 
octylphenol, but also of unexpected ER $\alpha$ ligands ( $n$-butylparaben, hydroxyl-methylbenzofuranone). High-resolution mass spectrometry results demonstrate that ER $\alpha$ affinity columns can be used for the isolation, purification, and identification of known as well as unknown estrogenic contaminants present in complex matrices. The third article of this special issue is also dealing with applied mathematical modeling applied to multi-biomarker approach for aquatic ecosystems assessment. However, exploration of large datasets by environmental managers represents a major gap for regulatory application of this tool. Sanchez et al. proposes to update calculation of the "Integrated Biological Response" described by Beliaeff and Burgeot (2002) to integrate recent knowledge on biomarker response. Indeed, it is recognized that a same biomarker can be induced or inhibited according to environmental exposure or physiological status of sentinel species. The novel index named "Integrated Biological Responses version 2 " is based on the reference deviation concept and allows a clear discrimination of sampling sites. It can be used in large monitoring programs with an absolute reference or in upstream/downstream studies in a pragmatic reference context.

A second set of articles presented in this special issue are dealing with effects of chemical or physical substances on different human and environmental endpoints. For instance, the article from Khorsi-Cauet et al. is studying the impact of the insecticide chlorpyrifos (CPF) on the mammalian digestive system by evaluating the effect of chronic, low-dose exposure to $\mathrm{CPF}$ on the composition of the gut microbiota in a simulator of the human intestinal microbial ecosystem, the SHIME $^{\circledR}$, compared to rats exposed in utero. These results for chronic, low-dose exposure to CPF in the $\mathrm{SHIME}^{\circledR}$ and in the rat were found to induce an imbalance in the microbial community, in particular, proliferation of subpopulations of potentially pathogenic bacteria and a decrease in the numbers of beneficial bacteria. In compliance with European guidelines, the use of the SHIME ${ }^{\circledR}$ in vitro tool would help to elucidate the final health effect of toxic agents. In their article, Pelletier et al. are dealing with effect of radio frequency electromagnetic field (RF-EMF) on the control of body energy balance in developing organisms despite the involvement of energy status in vital physiological functions. Then, the effects of chronic RF-EMF exposure $\left(900 \mathrm{MHz}, 1 \mathrm{Vm}^{-1}\right.$ ) on the main functions involved in the body energy homeostasis (feeding behavior, sleep and thermoregulatory processes) were examined. Most of the observed effects of RF-EMF exposure were dependent on air temperature. Exposure to RF-EMF appears to modify the functioning of vasomotor tone by acting peripherally through $\alpha$ adrenoceptors. The elicited vasoconstriction may restrict body cooling, whereas energy intake increases. These results show that RF-EMF exposure can induce energy-saving processes without strongly disturbing of the overall sleep pattern. The last article dealing with the effects of chemical and physical substances on different human and environmental endpoints is evidencing effect of in vivo chronic exposure to clotrimazole on zebrafish testis function. Baudiffier et al., 2012 previously showed that a 7-day exposure to clotrimazole induced the expression of genes related to steroidogenesis in the testes as a compensatory response, involving the activation of the Follicle stimulating hormone (Fsh)/Follicle stimulating hormone receptor (Fshr) pathway. In the present work, Brion et al. assess the effect of in vivo 21-day chronic exposure to clotrimazole $(30-197 \mu \mathrm{g} / \mathrm{L})$ on zebrafish testis function, i.e., spermatogenesis and androgen release. The chronic exposure led to an induction of steroidogenesis-related genes and fshr in the testes as well as $f \operatorname{sh} \beta$ in the pituitary. Moreover, increases of the gonadosomatic index and of the volume proportion of interstitial Leydig cells were observed in clotrimazoleexposed fish. In accordance with these histological observations, the circulating concentration of 11-Ketotestosterone had increased. Morphometric analysis of the testes did not show an effect of clotrimazole on meiotic (spermatocytes) or postmeiotic (spermatids and spermatozoa) stages, but we observed an increase in the number of type A spermatogonia, in agreement with an increase in mRNA levels of piwill, a specific molecular marker of type A spermatogonia. This study demonstrated that clotrimazole is able to affect testicular physiology and raised further concern about the impact of clotrimazole on reproduction.

In the last part of this special issue, articles are concentrating on technological tools designed to assess human and environmental impact of chemical and physical substances. For instance, Vranic et al. studied the uptake of fluorescently labeled $\mathrm{SiO} 2$ nanoparticles (NPs) of two different sizes (50 and $100 \mathrm{~nm}$ ) in two lung target cell types: human lung epithelial cells (NCI-H292) and mouse alveolar macrophages (MHS) in presence or absence of surfactant, a lung lining fluid that can potentially interact with NPs before they reach target cells. Internalization was shown to be time and dose dependent with a difference in the uptake efficacy between two cell types. In the light of these observations, authors conclude that flow cytometry gives more reliable results in studying NP internalization. Presence of surfactant is shown to diminish NP uptake in both cell types. In the same dynamic, Froment et al. postulate that few in vitro tests using a non-mammalian model such as bird have been explored to reproductive adverse effect study despite their advantages: the embryonic gonads of birds have a high plasticity of development sensitive to estrogen and sperm production is nearly two times faster than in rodents. In their 
work, authors have established an in vitro culture of germ cells and somatic cells from chicken postnatal testis and have evaluated the sensitivity against the endocrine disruptors: mono-(2-ethylhexyl) phthalate in comparison to previous studies using rodent and human models. Froment et al. conclude that the establishment of this postnatal testicular cells culture could be considered as an alternative method to in vivo experiments frequently used to evaluate impact on the terrestrial wildlife species. This method could be also complementary to mammal models, due to the limiting number of animals used and its elevated sensitivity. The last article presented in this special issue is dedicated to the presentation of a polar organic compound integrative sampler (POCIS) and a semipermeable membrane device (SPMD) passive sampler to assess the distribution of estrogenic, (anti-)androgenic, pregnane $\mathrm{X}$ receptor-like and dioxin-like activities between sediment and water compartments. Ait-Aissa et al. used these technologies in a river where sediment has been previously described as highly and multi-contaminated. The contamination evidenced a different distribution pattern of POCIS extracts and SPMD extracts activities between compartments. In order to establish whether the chemicals involved in these activities were similar between the compartments, the authors fractionated sediment, POCIS and SPMD extracts using a multi-step fractionation procedure. This highlighted differences in the nature of active chemicals between compartments. Altogether, these results support the need to consider different compartments in order to enhance exposure assessment.

Altogether, the articles presented in this special issue give a state of concepts, tools for predictive toxicology and ecotoxicology, as well as their potential used in concrete situation. Over scientific considerations, an important challenge remains validation even recognition of these concepts and tools.

\section{References}

Beliaeff B, Burgeot T (2002) Integrated biomarker response: A useful tool for ecological risk assessment. Environ Toxicol Chem 2 (6): 1316-22

Baudiffier D, Hinfray N, Vosges M, et al (2012) A critical role of follicle-stimulating hormone (Fsh in mediating the effect of clotrimazole on testicular steroidogenesis in adult zebrafish. Toxicology 298(1-3):30-9

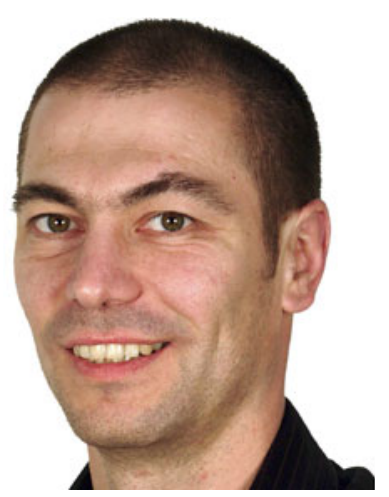

Dr. Emmanuel Lemazurier PhD in Endocrinology of Reproduction (2000) was a Visiting Researcher at the Prince Henry's Institute of Medical Research, Melbourne Australia. From 2001 to 2007 , he was conducting in INERIS several research projects in the field of endocrinedisrupting compounds. As a GLP Study Director, he was working with regulatory study plan in the field of toxicology. $\mathrm{He}$ also is very involved in the development of REACH-based new tools. Since 2008, Emmanuel Lemazurier is a project leader at the Scientific Division of INERIS and is involved in the strategic approach for chemical safety assessment. He is the General Secretary of the national network ANTIOPES from 2006 which aims to develop research for REACH implementation.

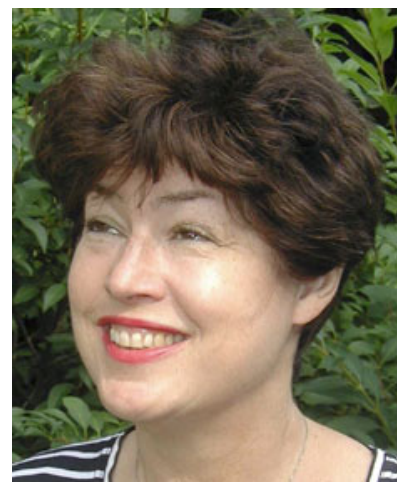

Francelyne Marano is Professor emeritus of Cellular Biology and Toxicology at University Paris Diderot where she has created the Laboratory of Cellular Physiology and Toxicology, now Molecular and Cellular Responses to Xenobiotics. She has actively participated to the development of cellular and molecular toxicology in France and in Europe during the last 20 years. She was the president of the French Society of Cellular Pharmacology and Toxicology (1999-2008) and copresident of the European Society of In Vitro Toxicology. She was the first president of the scientific committee of AFSSE (French Agency of Environmental Health). In 2009-2010, she has chaired a national committee at the Ministry of Research to define the national strategies of researches in toxicology and ecotoxicology. She is a member of the French National Council for Public Health (HCSP) and chairs the committees on the effects of nanotechnology on health and on emerging risks. She is the president of ANTIOPES network.

Her research studies are in the field of cellular and molecular toxicology: studies on the mechanisms of effects of atmospheric pollutants and specially the particulate pollutants and nanoparticles on the human lung, development of alternative methods. She has authored more than 150 publications and was scientific editor of several books.

She was expert in several national and European scientific committees on environmental toxicology and health (PRIMEQUAL, ANR SEST and CES, SCHENIR and SCCP, DG SANCO) and now member or copresident of the scientific councils of two famous national institutes: AgroParistech and INERIS. She has participated to the "Grenelle de l'Environnement" (2008-2009) nominated as an expert in the field of environmental health and toxicology by the French Ministries of Environment and of Research. 